\title{
Efecto del compost de residuos orgánicos domiciliares, vegetales y estiércol en el crecimiento de lechuga
}

\section{Effect of organic residue compost made from household waste, plant residues and manure on the growth of lettuce}

DAIANNI ARIANE DA COSTA FERREIRA'

NILDO DA SILVA DIAS'

ANA KALINE DA COSTA FERREIRA'

CYBELLE BARBOSA E LIMA VASCONCELOS'

FRANCISCO SOUTO DE SOUSA JUNIOR ${ }^{2}$

VANIA CHRISTINA NASCIMENTO PORTO ${ }^{1}$

CLEYTON DOS SANTOS FERNANDES2,4

MANUEL ANTONIO NAVARRO VÁSOUEZ ${ }^{3}$

Estudio con lechuga orgánica.

Foto: D.A.d.C. Ferreira

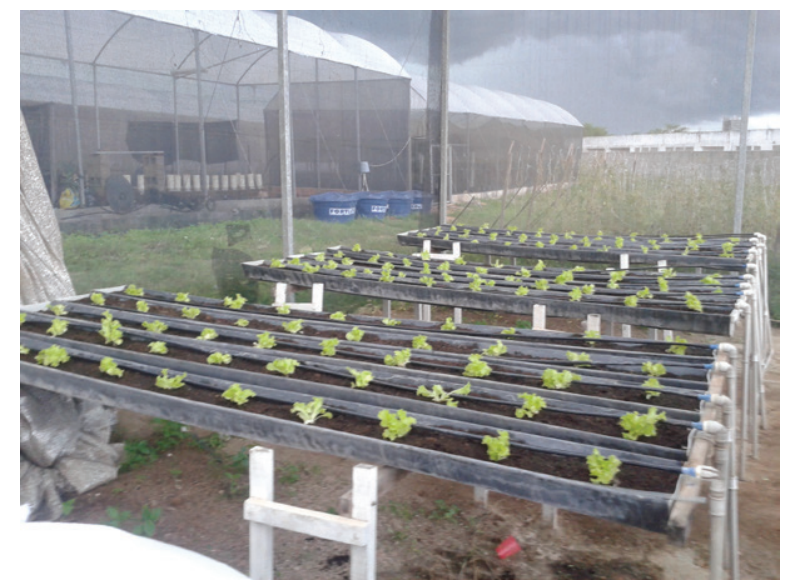

\section{RESUMEN}

El compostaje se presenta como una alternativa para reciclar residuos sólidos orgánicos biodegradables, transformarlos en fertilizantes para la agricultura y evitar su deposición inadecuada en rellenos sanitarios. De esta forma, el objetivo del presente estudio fue evaluar la calidad físico-química y microbiológica de un compost producido a partir de residuos orgánicos y su desempeño en el desarrollo y productividad del cultivo de lechuga crespa (Lactuca sativa L.), en condiciones de invernadero. La materia prima del compostaje fueron residuos orgánicos domiciliares (restos de alimentos) y estiércol de bovino en proporción 1:1. Para la construcción de los montículos de compost, los residuos orgánicos fueron mezclados con podas de árboles triturados. Diferentes concentraciones de compost madurado, 20,40,60,80 y 100\%, fueron incluidos en el sustrato de lechuga en un diseño de bloques al azar con tres repeticiones. Los resultados mostraron que el compost orgánico producido presentó características físico-químicas y microbiológicas dentro de los rangos de utilización agronómica y su adición en el sustrato de fibra de coco, favoreció la producción de lechuga, promoviendo un incremento de $63 \%$ en la altura de plantas y $75 \%$ en el número de hojas.

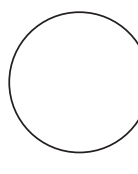

Palabras clave adicionales: Lactuca sativa L., fertilización, agricultura urbana, manejo de residuos, producción de hortalizas.

Universidade Federal Rural do Semi-Árido, Campus Oeste, Mossoro-RN (Brasil). ORCID Ferreira, D.A.d.C.: 0000-0002-9878-061X; ORCID Dias, N.d.S.: 0000-0002-1276-5444; ORCID Ferreira, A.K.d.C.: 0000-0001-7739-574X; ORCID Vasconcelos, C.B.e.L.: 0000-0003-1463-1148; ORCID Porto, V.C.N.: 0000-0002-0352-0162

2 Universidade Federal Rural do Semi-Árido, Campus Angicos, Angicos-RN (Brasil). ORCID Sousa Júnior, F.S.d.: 00000003-2599-0023; ORCID Fernandes, C.d.S.: 0000-0002-9465-2637

3 Instituto Federal de Educação Ciência e Tecnologia do Ceara (IFCE), Crato-CE (Brasil). ORCID Vásquez, M.A.N.: 0000-0001-6207-1603

4 Autor para correspondencia. cleyton1959@hotmail.com 


\section{ABSTRACT}

Composting is an alternative for recycling biodegradable organic solid wastes, turning them into fertilizers for agriculture and avoiding improper disposal in landfills. Thus, the objective of the present study was to evaluate the physical-chemical and microbiological quality of a compound produced from organic residues and its performance in the development and productivity of a crisp lettuce cultivar (Lactuca sativa L.), in greenhouse conditions. For the compost, household waste (food residue) and cattle manure were used at a 1:1 ratio. For the construction of the compost piles, the organic residues were mixed with wood chips. Different concentrations of the mature compost $(20,40,60,80$, and $100 \%$ compost) were evaluated in the lettuce cultivation substrate in a randomized block design, with three replicates. The results showed that the organic compost had physical-chemical and microbiological characteristics within agronomic use patterns that, when added to the coconut fiber substrate, favored lettuce production, promoting a $63 \%$ increase in plant height and $75 \%$ increase in the number of leaves.

Additional key words: Lactuca sativa L., fertilization, urban agriculture, waste management, vegetable production.

Fecha de recepción: 27-03-2018 Aprobado para publicación: 30-05-2018

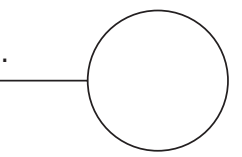

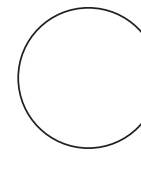

Buena parte de los residuos sólidos producidos actualmente no poseen un destino sanitaria y ambiental adecuado. A pesar de avances en los últimos años, los residuos todavía son depositados en basurales a cielo abierto en más de la mitad de los municipios brasileros (Gouveia, 2012); de estos residuos más del 50\% son orgánicos (Maragno et al., 2007).

El gran problema ambiental de la deposición de estos residuos en basurales y rellenos sanitarios son la elevada producción de líquidos (lixiviados) que puede fácilmente infiltrarse y contaminar el suelo, además, de las aguas del nivel freático y subterráneas. Con la expansión y alta densidad de las urbes, estos problemas tienen a empeorar teniendo en cuenta que la mayoría de las ciudades brasileras no proyectan el ritmo acelerado de su crecimiento con infraestructura sanitaria adecuada (Rezende et al., 2013; Cotta et al., 2015).

La gestión de residuos sólidos orgánicos es un problema que enfrenta una gran parte de los países en desarrollo, debido al ritmo acelerado de crecimiento de la población, de la actividad económica y de la urbanización e industrialización que intensifican el proceso de generación de esos residuos (Srivastava et al., 2015; Kumar et al., 2017; Butu y Mshelia, 2014).

El gran desafío de las instituciones gubernamentales es garantir la destinación adecuada de los residuos orgánicos de forma que evite impactos negativos al medio ambiente, promoviendo la implantación de un sistema de manejo diferenciado y un tratamiento de residuos sólidos que promueva la segregación de las fuentes generadoras, minimice los impactos ambientales y maximice los beneficios sociales y económicos para la población (Albertin et al., 2010). En algunos países desarrollados, como Polonia, la gestión de residuos sólidos orgánicos es considerado una de las actividades más importantes de la protección ambiental (Boer et al., 2010).

El reciclaje de residuos orgánicos por medio de proceso de compostaje se viene constituyendo como el principal medio de tratamiento ante la gran cantidad de residuos que llegan a los rellenos sanitarios (Maragno et al., 2007), donde la masa de residuo es tratada $y$ transformada en un nuevo producto, mediadas por microorganismos en abono orgánico (Pereira Neto, 2007; Yu y Huang, 2009; Lesteur et al., 2010; Li et al., 2013) y que puede ser utilizado como fertilizante en la agricultura (Pedrosa et al., 2013; Dores-Silva et al., 2013), garantizando así la deposición segura de estos materiales en el ambiente (Figueiredo y Tanamati, 2010; Montemurro et al., 2010) y reduciendo el uso de fertilizantes convencionales.

En este contexto, el objetivo de este trabajo fue evaluar las características químicas, físicas y biológicas de un compost producido a partir de residuos orgánicos domiciliares y vegetales, y su desempeño en el desarrollo y productividad del cultivo de lechuga crespa, cultivada en invernadero. 


\section{MATERIALES Y MÉTODOS}

La investigación fue desarrollada entre agosto de 2015 y marzo de 2016. Se realizaron dos etapas, la primera en la producción de compost y la segunda el cultivo de lechuga con el compost generado en condiciones de invernadero.

\section{Producción de compost}

La primera etapa de la investigación fue desarrollada en campo abierto en el municipio de Mossoró, Rio Grande do Norte (en Asociación Comunitaria Reciclando para la Vida, ACREVI), ubicado a 5¹4'9" S y 37०18'59' W, a 18 msnm.

Según la clasificación de Köppen, el clima de la región es de tipo seco y muy caliente (BSwh1). La precipitación pluviométrica es bastante irregular con media anual de $673,9 \mathrm{~mm}$ y una estación seca que generalmente comprende el período de junio a enero y la estación lluviosa entre febrero y mayo. La temperatura media anual de $27^{\circ} \mathrm{C}$ y la humedad relativa del aire presenta una media de 68,9\% (Carmo Filho y Oliveira, 1995). En el momento del compostaje, entre septiembre y diciembre de 2015, la temperatura media registrada fue de $28,4^{\circ} \mathrm{C}$ y la humedad media de 59,5\% (Fig. 1).

El proceso de compostaje fue realizado con montículos directamente sobre el suelo teniendo como materia prima tres materiales: residuos orgánicos domiciliares (restos de alimentos), residuos vegetales (podas de árboles) y estiércol bovino.
Los residuos alimenticios fueron obtenidos durante la colecta selectiva realizada por la ACREVI en el restaurante popular "Barriga Cheia" del municipio de Mossoró. Los residuos vegetales fueron colectados por el servicio de limpieza urbana del municipio de Mossoró y, posteriormente, fueron triturados con una desbrozadora marca Vermeer (modelo BC1000XL de 85 hp, Vermeer Corporation, Pella, IA, USA). El estiércol bovino fue obtenido del área de bovinos de la Universidade Federal Rural do Semi-Árido (UFERSA, Mossoró-RN, Brasil).

Para la construcción de los montículos de compostaje se siguió la metodología "Windrow" (Pereira Neto, 2007), que tuvieron la forma cónico de dimensiones $1,60 \mathrm{~m}$ de altura y 2,00 $\mathrm{m}$ de diámetro y peso total aproximado de $550 \mathrm{~kg}$. Se aplicaron los residuos en capas alternas de $30 \mathrm{~cm}$ de residuo vegetal, $5 \mathrm{~cm}$ de residuos domiciliares y $5 \mathrm{~cm}$ de estiércol bovino hasta la altura deseada. Después de cada capa, el montículo era regado de forma uniforme, con agua proveniente de la Companhia de Águas e Esgotos do Rio Grande do Norte (CAERN) (Natal-RN, Brasil).

La mezcla o revuelta de los montículos para su aireación fue realizada de forma manual cada $3 \mathrm{~d}$ después de iniciar el compostaje y luego cada 10 d. Se aplicó el riego manual y diariamente. Para disminuir la evaporación del agua en el montículo se cubrió con hojas secas de coquero.

Para verificar la maduración del compostaje fueron realizadas pruebas de mano y de bola descritos por Kiehl (2002). Además, en el momento del muestreo

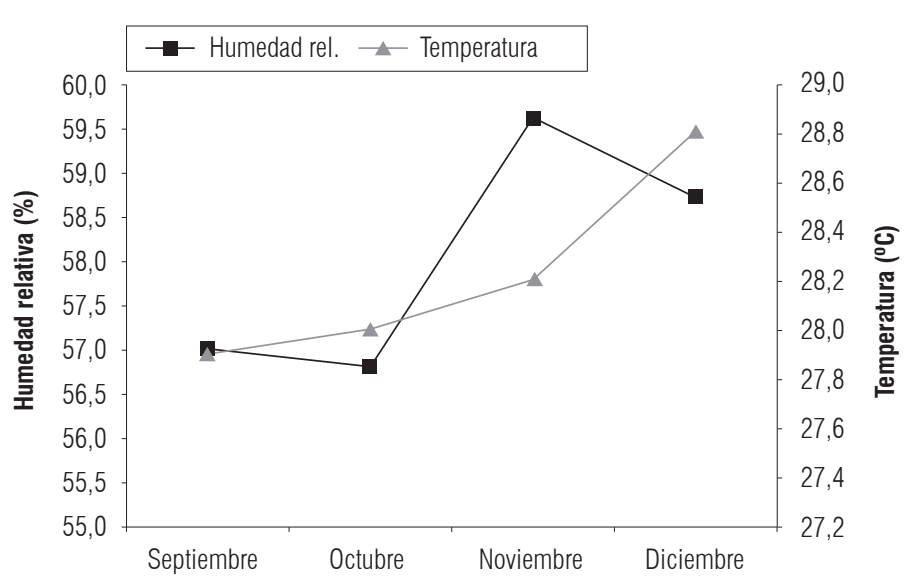

Figura 1. Humedad relativa y temperatura media del aire en el momento de realizar el compostaje. Fuente: Instituto Nacional de Meteorología (INMET, 2016). 
para los análisis de laboratorio se aplicó el método de independencia de la muestra. Las muestras fueron acondicionadas en sacos plásticos, identificadas y enviadas al Laboratorio de Agua, Suelo y Planta (LASAP) de la UFERSA para la determinación de las propiedades físico-químicas (Embrapa, 2009) y microbiológicas (APHA, 2012).

Se determinaron las propiedades químicas $\mathrm{P}, \mathrm{K}, \mathrm{Ca}$, $\mathrm{Mg}, \mathrm{Fe}, \mathrm{Cu}, \mathrm{Mn}, \mathrm{Zn}, \mathrm{Ni}, \mathrm{Cd}$ y $\mathrm{Pb}$ en dos momentos: una después de la construcción del montículo y otra al final del proceso de compostaje. A los 0, 30, 60 y 90 d después de la construcción de los montículos se realizó la medición del carbono orgánico total, sustancias húmicas, nitrógeno total y relación $\mathrm{C} / \mathrm{N}$.

La caracterización microbiológica del compost fue determinada por conteo de microrganismos a través del método del número más probable (NMP). Los análisis fueron realizados en el Laboratorio de Inspección de Productos de Origen Animal (LIPOA) de la UFERSA. Inicialmente, se evaluó la presencia de coliformes totales y termotolerantes. Posteriormente, la detección de Salmonella sp. fue realizada por el enriquecimiento de los medios líquidos selectivos Rappaport vassiliadis, selenito-cistina y tetrationato.

Al finalizar el proceso de compostaje con la degradación de la mayor parte de los residuos adicionados al montículo, el material fue tamizado para la separación del material grueso, y el compuesto humificado fue acondicionado en sacos plásticos para su transporte.

\section{Producción de lechuga}

La segunda etapa del trabajo fue desarrollada entre febrero y marzo de 2016 en la UFERSA, Campus Oeste, en Mossoró-RN, localizada en las coordenadas geográficas $5^{\circ} 11^{\prime}$ S y $37^{\circ} 20^{\prime} \mathrm{W}$ a $18 \mathrm{msnm}$. El diseño experimental utilizado para evaluar la viabilidad del compost en la producción de lechuga fue bloques al azar con 5 tratamientos y 3 repeticiones. Los tratamientos consistieron en diferentes dosis del compost preparado de la primera etapa en proporción de 20, 40, 60,80 y $100 \%$, mezclado con el sustrato de fibra de coco. La unidad experimental se formó por 10 plantas.

Se empleó lechuga var. Vera, tipo crespa, producida en bandejas de poliestireno expandido de 128 celdas con humus de lombriz. Se realizó el trasplante a los $23 \mathrm{~d}$ después de la siembra cuando presentaban entre 5 a 6 $\mathrm{cm}$ de altura y 5 hojas definitivas.

El cultivo de lechuga se realizó en canaletas de PVC tipo trapecio con $3 \mathrm{~m}$ de largo, 0,2 $\mathrm{m}$ de ancho y distancia entre plantas de $0,3 \mathrm{~m}$. Las canaletas fueron fijadas a un suporte de madera de $0,90 \mathrm{~m}$ del suelo con pendiente del $3 \%$; se realizaron perforaciones cada 0,5 m para drenar el exceso de agua.

El riego se aplicó diariamente en dos momentos, al inicio de la mañana y final de la tarde. Se implementó un sistema de riego por goteo con emisores distanciados cada $0,3 \mathrm{~m}$ y un caudal de $2,5 \mathrm{~L} \mathrm{~h}^{-1}$. El agua utilizada provenía del pozo de abastecimiento de la UFERSA (Tab. 1).

A los 35 días después del trasplante (dat), fueron evaluados los parámetros de crecimiento, macro y micronutrientes y parámetros microbiológicos en las plantas de lechuga. Los parámetros de crecimiento evaluados fueron: número de hojas por planta (NHP), diámetro del tallo (DT), altura de la parte aérea (APA), masa fresca de la parte aérea (MFPA), masa seca de la parte aérea (MSPA), masa fresca de la raíz (MFR) y masa seca de la raíz (MSR).

Las plantas fueron secas en estufa con circulación de aire forzado a temperatura de $65^{\circ} \mathrm{C}$, hasta presentar masa constante y, en seguida, fue determinada la producción de masa seca. Después de pesadas, las muestras fueron trituradas y acondicionadas en recipientes herméticamente cerrados para realizar los análisis de nutrientes ( $\mathrm{N}, \mathrm{Ca}, \mathrm{Mg}, \mathrm{Cu}, \mathrm{Zn}, \mathrm{Fe}, \mathrm{Mn}$ ), utilizando espectroscopio de absorción atómica modelo AA 240

Tabla 1. Caracterización físico-química de agua de riego utilizada en el cultivo de lechuga producida con compost de residuos vegetales, orgánicos y estiércol bovino.

\begin{tabular}{|c|c|c|c|c|c|c|c|c|c|}
\hline \multirow{2}{*}{ Fuente } & \multirow{2}{*}{$\mathrm{CE}\left(\mathrm{dS} \mathrm{m} \mathrm{m}^{-1}\right)$} & \multirow{2}{*}{$\mathrm{pH}$} & $\mathrm{Ca}^{2+}$ & $\mathrm{Mg}^{2+}$ & $\mathrm{Na}^{+}$ & $\mathrm{Cl}$ & $\mathrm{CO}_{3}{ }^{2}$ & $\mathrm{HCO}^{3-}$ & $\mathrm{RAS}$ \\
\cline { 4 - 10 } & & \multicolumn{6}{|c|}{$\mathrm{mmol}_{\mathrm{c}} \mathrm{L}^{-1}$} & \multicolumn{2}{|c|}{$\mathrm{mg} \mathrm{L}^{-1}$} \\
\hline $\mathrm{PA}$ & 0,55 & 7,5 & 1,0 & 0,9 & 4,44 & 2,4 & 0,7 & 3,4 & 4,6 \\
\hline
\end{tabular}

PA: pozo de abastecimiento; CE: conductividad eléctrica; $\mathrm{CO}_{3}^{2}$ : carbonato; $\mathrm{HCO}_{3}$ : bicarbonato; RAS: reacción de adsorción de sódio. 
FS (Agilent, Technologies, Santa Clara, CA, USA). Las concentraciones de los macronutrientes $\mathrm{P}$ y $\mathrm{K}$ fueron determinadas por calorimetría, utilizándose espectrofotómetro de llama, modelo DR-62 (Digimed, São Paulo-SP, Brasil).

El análisis microbiológico fue realizado en el Laboratório de Inspeção de Produtos de Origem Animal (LIPOA) de la UFERSA, por el método de blanqueamiento en superficie, de acuerdo con la metodología descrita en el Compenduim of Methods for the Microbiological Examination of Foods de la APHA (American Public Health Association, Washington, DC, USA).

Los datos obtenidos en el experimento fueron sometidos a un análisis de variancia empleando la prueba F y en caso de significancia la prueba de Tukey al 1 y $5 \%$ de probabilidad. Además, se realizó un análisis de regresión para los datos cuantitativos, utilizando el software ASSISTAT 7.7.

\section{RESULTADOS}

\section{Cualidad nutricional del compost}

El análisis de la materia prima utilizada en el compostaje (residuos sólidos orgánicos domiciliares, residuos vegetales y estiércol bovino), presentó la mayor concentración de calcio, además, el hierro, manganeso y zinc (Tab. 2).

Tabla 2. Macro y micronutrientes en la materia prima (residuos orgánicos y vegetales + estiércol bovino) al inicio de compost.

\begin{tabular}{|c|c|c|c|}
\hline Macronutrientes & $\begin{array}{c}\text { Concentración } \\
\left(\mathrm{g} \mathrm{kg}^{-1}\right)\end{array}$ & Micronutrientes & $\begin{array}{c}\text { Concentración } \\
\left(\mathrm{mg} \mathrm{kg}^{-1}\right)\end{array}$ \\
\hline $\mathrm{N}$ & 0,74 & $\mathrm{Fe}$ & $1.345,00$ \\
\hline $\mathrm{P}$ & 0,75 & $\mathrm{Cu}$ & 5,70 \\
\hline $\mathrm{K}$ & 5,25 & $\mathrm{Mn}$ & 31,45 \\
\hline $\mathrm{Ca}$ & 19,71 & $\mathrm{Zn}$ & 26,35 \\
\hline $\mathrm{Mg}$ & 2,40 & $\mathrm{Ni}$ & 2,80 \\
\hline & & $\mathrm{Cd}$ & 0,50 \\
\hline & & $\mathrm{Pb}$ & 9,50 \\
\hline
\end{tabular}

La concentración de $\mathrm{N}$ fue mayor en el compost estabilizado que al inicio del compostaje (materias primas); para los demás macronutrientes la concentración disminuyó durante el proceso. La concentración de los micronutrientes Fe, Mn y Zn, el proceso de compostaje aumentó en comparación de la materia prima. Los contaminantes químicos como $\mathrm{Cd}$ y $\mathrm{Pb}$ se redujeron y se elevaron los niveles de $\mathrm{Ni}$ (Tab. 2 y 3 ).

Tabla 3. Metales pesados, macro y micronutrientes en el compost estabilizado con residuos orgánicos domiciliarios.

\begin{tabular}{|c|c|c|c|c|c|c|c|}
\hline \multicolumn{4}{|c|}{$\begin{array}{c}\text { Macro y micronutrientes } \\
\left(\mathrm{mg} \mathrm{kg}^{-1}\right)\end{array}$} & \multicolumn{4}{c|}{$\begin{array}{c}\text { Metales pesados } \\
\left(\mathrm{mg} \mathrm{kg}^{-1}\right)\end{array}$} \\
\hline $\mathrm{N}$ & $\mathrm{Fe}$ & $\mathrm{Cu}$ & $\mathrm{Mn}$ & $\mathrm{Zn}$ & $\mathrm{Ni}$ & $\mathrm{Cd}$ & $\mathrm{Pb}$ \\
\hline 0,83 & $1.826,0$ & 4,80 & 47,75 & 41,90 & 5,25 & 0,15 & 0,50 \\
\hline
\end{tabular}

La evolución del carbono orgánico total (COT) mostro decrecimiento a lo largo del proceso de compostaje (Fig. 2A). El nitrógeno total presentó pequeña reducción en la concentración en los primeros $30 \mathrm{~d}$ del proceso de compostaje manteniéndose constante durante todo el proceso de compostaje (Fig. 2B). El fraccionamiento de la materia orgánica durante el periodo de estabilización del compost (Tab. 4), demuestra que las sustancias húmicas están presentes en la siguiente orden de concentración: humina $>$ ácidos fúlvicos $>$ ácidos húmicos. La relación $\mathrm{C} / \mathrm{N}$ inicia con una proporción 26/1 y finaliza en 12/1 (Fig. 3).

\begin{tabular}{|c|c|c|c|}
\hline \multicolumn{4}{|c|}{ Fracciones orgánicas } \\
\hline Período & $\mathrm{C}-\mathrm{AH}$ & C-AF & $\mathrm{H}$ \\
\hline (d) & \multicolumn{3}{|c|}{$\left(\right.$ dag $\left.\mathrm{kg}^{-1}\right)$} \\
\hline 0 & 2,01 & 2,38 & 29,36 \\
\hline 30 & 0,59 & 0,62 & 18,44 \\
\hline 60 & 0,28 & 0,34 & 15,66 \\
\hline 90 & 0,27 & 0,21 & 13,79 \\
\hline$\%$ final & 1,9 & 1,5 & 96,6 \\
\hline
\end{tabular}

dag: decagramo

La estabilización del compost permitió que en los análisis microbiológicos se evidenciara la eliminación de los microrganismos patogénicos, coliformes totales, termotolerantes y Salmonelle durante el compostaje; los valores encontrados estuvieron por debajo del límite detectado, con cifras menores a 0,3; 0,0 y negativo, respectivamente.

\section{Producción de lechuga}

El análisis de variancia para las características evaluadas en la planta de lechuga, permitió identificar 

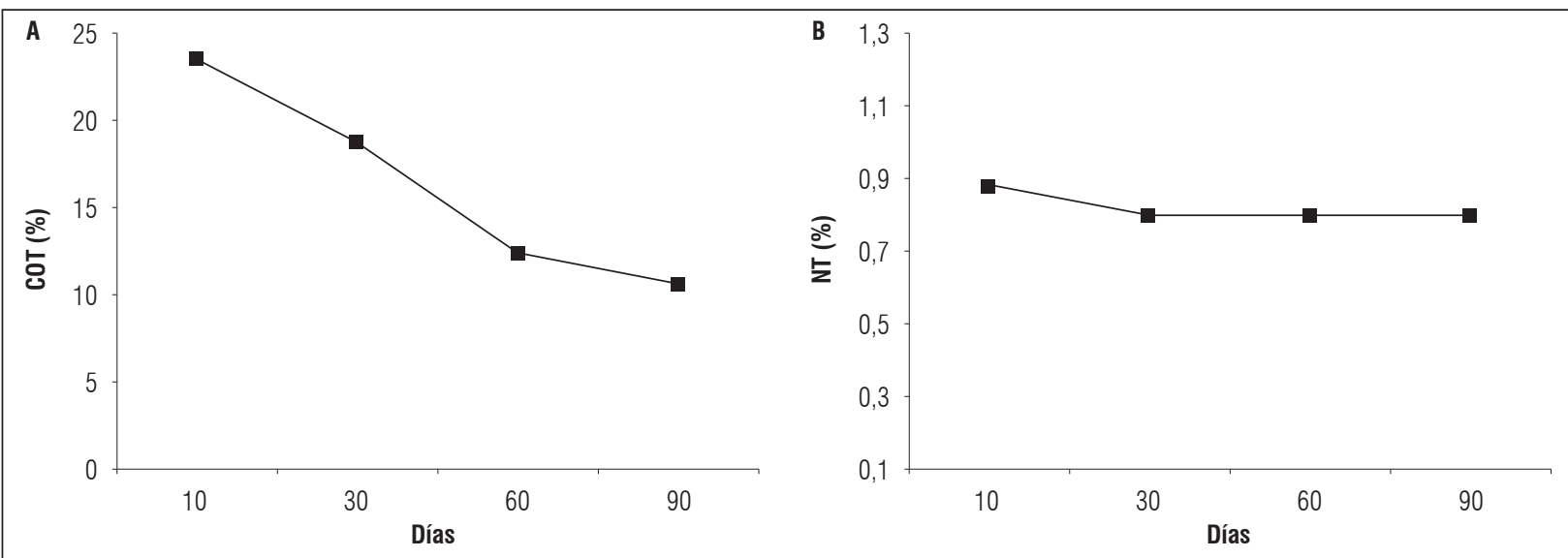

Figura 2. Concentración de carbono orgánico total (COT) (A) y nitrógeno total (NT) (B) en el montículo de compost con residuos orgánicos urbano a lo largo de $90 \mathrm{~d}$.

diferencias significativas en las variables altura de plantas (AP) y número de hojas (NF) en los tratamientos aplicados (Tab. 5).

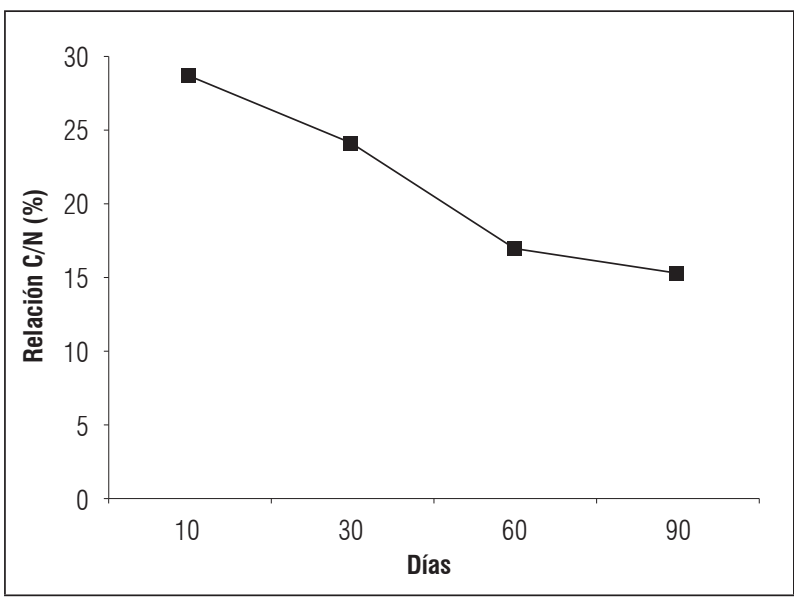

Figura 3. Relación carbono/nitrógeno (C/N) en el compostaje de residuos orgánicos domiciliarios.
La altura de planta y el número de hojas aumentaron linealmente con las dosis crecientes de compostaje (Fig. 4), donde la dosis más alta de compost (100\%) presentó los mejores valores para estas variables.

En la tabla 6 se presentan los valores medios para las variables DT, MFPA, MSPA, MFR y MSR que no presentaron diferencias significativas entre las diferentes dosis de compost.

Con respecto al análisis de variancia para las concentraciones medias de nutrientes en las plantas de lechuga, los tratamientos presentaron diferencias significativas al nivel de $1 \%$ de probabilidad para los niveles de nitrógeno, calcio y magnesio (Tab. 7).

Las concentraciones de $\mathrm{N}$, Ca y $\mathrm{Mg}$ se elevaron en las plantas de lechuga conforme aumentaba la dosis de compostaje (Tab. 8). Se encontraron mayores contenido de $\mathrm{N}$ en las dosis 80 y $100 \%$ con diferencias significativas con los demás tratamientos. Con respecto a los contenidos de Ca y Mg, sobresalieron las dosis

Tabla 5. Análisis de variancia para componentes de crecimiento y desarrollo de lechuga, cultivadas con diferentes dosis de compostaje de residuos orgánicos domiciliares.

\begin{tabular}{|l|c|c|c|c|c|c|c|c|}
\hline Fuente de variación & GL & AP & DT & NF & MFPA & MSPA & MFR & MSR \\
\hline Bloques & 2 & $2,57^{\text {ns }}$ & $0,1^{\text {ns }}$ & $2,24^{\text {ns }}$ & $58,58^{\text {ns }}$ & $0,11^{\text {ns }}$ & $1,83^{\text {ns }}$ & $0,05^{\text {ns }}$ \\
\hline Tratamientos & 4 & $6,7^{*}$ & $0,87^{\text {ns }}$ & $9,87^{* *}$ & $122,23^{\text {ns }}$ & $0,41^{\text {ns }}$ & $7,26^{\text {ns }}$ & $0,02^{\text {ns }}$ \\
\hline Residuo & 8 & 1,73 & 1,73 & 1,24 & 32,37 & 0,22 & 4,44 & 0,54 \\
\hline CV \% & & 13,52 & 25,24 & 10,71 & 52,22 & 43,53 & 39,10 & 45,74 \\
\hline
\end{tabular}

AP: altura de plantas, DT: diámetro de tallo (DH), NH: número de hojas, MFPA: masa fresca de la parte aérea, MSPA: masa seca de la parte aérea, MFR: masa fresca de la raíz, MSR: masa seca de la raíz.

** significancia a $P \leq 0,01,{ }^{*}$ significancia a $P \leq 0,05$, ns no significativo con la prueba F. GL: grados de libertad; CV: coeficiente de variación. 
Tabla 6. Componentes de crecimiento y desarrollo de lechuga, cultivadas con diferentes dosis de compostaje de residuos orgánicos domiciliares.

\begin{tabular}{|c|c|c|c|c|c|}
\hline Dosis de compost & DT & MFPA & MSPA & MFRAIZ & MSRAIZ \\
\hline$(\%)$ & $(\mathrm{mm})$ & \multicolumn{4}{|c|}{ (g/planta) } \\
\hline 20 & $4,63 \mathrm{a}$ & $5,62 \mathrm{a}$ & $0,84 \mathrm{a}$ & $4,19 \mathrm{a}$ & 0,58 a \\
\hline 40 & $4,78 \mathrm{a}$ & $5,46 a$ & $0,68 \mathrm{a}$ & $3,43 \mathrm{a}$ & $0,42 a$ \\
\hline 60 & $5,23 a$ & $8,74 a$ & $0,94 \mathrm{a}$ & $5,76 a$ & $0,45 a$ \\
\hline 80 & $5,40 \mathrm{a}$ & $14,30 \mathrm{a}$ & $1,28 \mathrm{a}$ & $7,24 \mathrm{a}$ & $0,61 \mathrm{a}$ \\
\hline 100 & $5,98 \mathrm{a}$ & $20,35 \mathrm{a}$ & $1,61 \mathrm{a}$ & $6,23 \mathrm{a}$ & $0,49 a$ \\
\hline DMS & 3,71 & 16,06 & 1,31 & 5,94 & 0,65 \\
\hline
\end{tabular}

AP: altura de plantas, DT: diámetro de tallo (DH), NH: número de hojas, MFPA: masa fresca de la parte aérea, MSPA: masa seca de la parte aérea, MFR: masa fresca de la raíz, MSR: masa seca de la raíz

Promedios con letras distintas indican diferencia significativa según la prueba de Tukey $(P \leq 0,05)$; DMS: diferencia mínima significativa.

Tabla 7. Análisis de variancia para la concentración de nutrientes en la planta de lechuga, cultivada con diferentes dosis de compostaje de residuos orgánicos domiciliarios.

\begin{tabular}{|l|c|c|c|c|c|c|c|c|c|c|}
\hline Fuente de variación & $\mathrm{GL}$ & $\mathrm{N}$ & $\mathrm{P}$ & $\mathrm{K}$ & $\mathrm{Ca}$ & $\mathrm{Mg}$ & $\mathrm{Cu}$ & $\mathrm{Mn}$ & $\mathrm{Fe}$ & $\mathrm{Zn}$ \\
\hline Bloques & 2 & $0,50^{\text {ns }}$ & $0,11^{\text {ns }}$ & $0,29^{\text {ns }}$ & $3,62^{\text {ns }}$ & $0,45^{*}$ & $38,86^{* *}$ & $18,58^{\text {ns }}$ & $509,07^{\text {ns }}$ & $219,38^{\text {ns }}$ \\
\hline Tratamientos & 4 & $160,64^{* *}$ & $0,02^{\text {ns }}$ & $0,13^{\text {ns }}$ & $26,59^{* *}$ & $0,76^{* *}$ & $1,57^{\text {ns }}$ & $260,39^{\text {ns }}$ & $546,16^{\text {ns }}$ & $58,13^{\text {ns }}$ \\
\hline Residuo & 8 & 3,26 & 0,05 & 0,09 & 2,67 & 0,09 & 1,75 & 154,31 & 347,03 & 98,26 \\
\hline CV\% & & 8,17 & 13,87 & 10,12 & 19,81 & 13,31 & 46,93 & 29,74 & 33,40 & 17,38 \\
\hline
\end{tabular}

** significancia a $P \leq 0,01,{ }^{*}$ significancia a $P \leq 0,05$ y ${ }^{\text {ns }}$ no significativo con la prueba F. GL: grados de libertad; CV: coeficiente de variación.
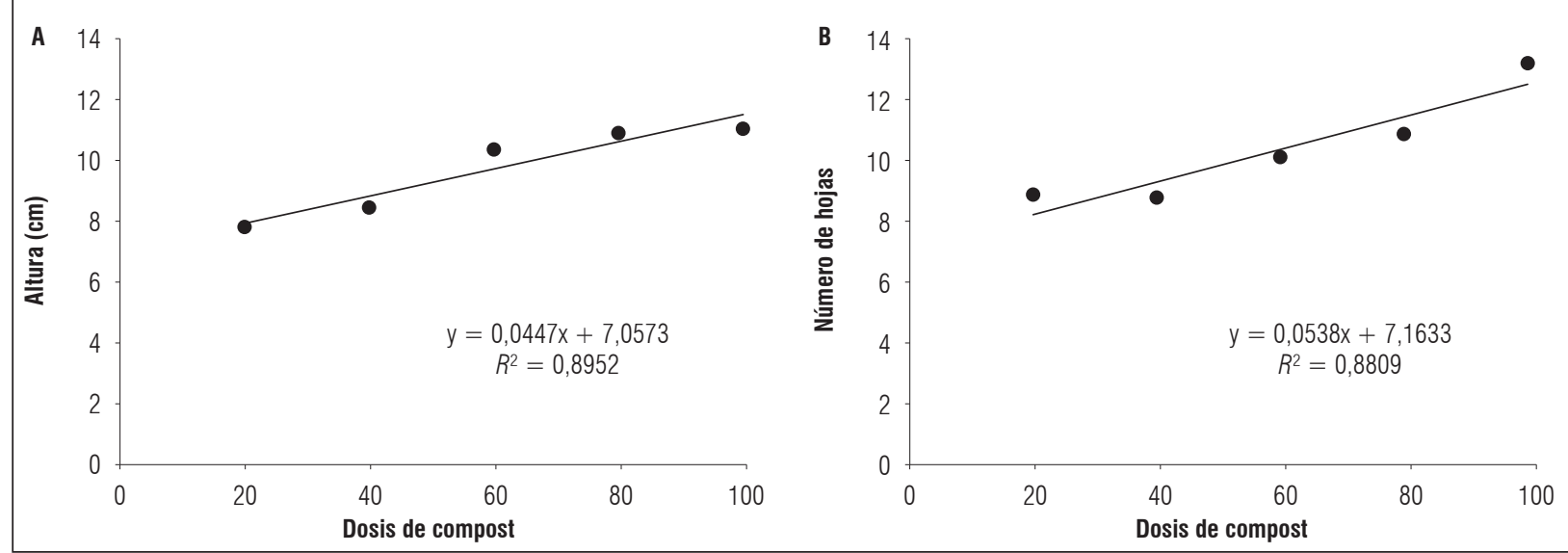

Figura 4. Altura de planta (A) y número de hojas (B) en plantas de lechuga cultivadas con diferentes dosis de compostaje con residuos orgánicos domiciliarios.

60, 80 y $100 \%$ sin diferencias entre estas, pero sí con las demás.

Se encontraron coliformes totales en muestras de humedad de las hojas de lechuga (al natural), en recuentos a $45^{\circ} \mathrm{C}$ para los diferentes tratamientos. Se constataron valores de 20, 35, 40, 50 y $70 \mathrm{UFC/g}$ en las lechugas producidas con las dosis de 20, 40, 60, 80 y $100 \%$ de compost, respectivamente. No obstante, estos resultados se encuentran dentro de los límites establecidos en la Resolución - RDC 12/2001 de la Agencia Nacional de Vigilancia Sanitaria (ANVISA), 
Tabla 8. Macro y micronutrientes foliares en plantas de lechuga establecidas en diferentes dosis de compostaje de residuos orgánicos domiciliarios.

\begin{tabular}{|c|c|c|c|c|c|c|c|c|c|c|}
\hline \multirow{2}{*}{$\begin{array}{c}\text { Dosis de } \\
\begin{array}{c}\text { compos } \\
(\%)\end{array}\end{array}$} & $\mathbf{N}$ & $\mathbf{P}$ & $\mathbf{K}$ & $\mathrm{Ca}$ & $\mathbf{M g}$ & $\mathrm{Cu}$ & \multicolumn{5}{|c|}{$\mathrm{Mn}$} & $\mathrm{Fe}$ & $\mathrm{Zn}$ \\
\cline { 2 - 11 } & \multicolumn{9}{|c|}{$\left(\mathrm{mg} \mathrm{kg}^{-1}\right)$} \\
\hline 20 & $14,1 \mathrm{c}$ & $1,62 \mathrm{a}$ & $2,97 \mathrm{a}$ & $5,12 \mathrm{~b}$ & $1,97 \mathrm{~b}$ & $2,33 \mathrm{a}$ & $38,61 \mathrm{a}$ & $56,16 \mathrm{a}$ & $52,22 \mathrm{a}$ \\
\hline 40 & $15,9 \mathrm{c}$ & $1,46 \mathrm{a}$ & $2,79 \mathrm{a}$ & $5,31 \mathrm{~b}$ & $1,73 \mathrm{~b}$ & $2,31 \mathrm{a}$ & $37,63 \mathrm{a}$ & $36,95 \mathrm{a}$ & $54,98 \mathrm{a}$ \\
\hline 60 & $22,07 \mathrm{~b}$ & $1,64 \mathrm{a}$ & $3,29 \mathrm{a}$ & $9,23 \mathrm{ab}$ & $2,35 \mathrm{ab}$ & $2,23 \mathrm{a}$ & $30,27 \mathrm{a}$ & $49,58 \mathrm{a}$ & $60,56 \mathrm{a}$ \\
\hline 80 & $26,93 \mathrm{ab}$ & $1,62 \mathrm{a}$ & $2,80 \mathrm{a}$ & $9,47 \mathrm{ab}$ & $2,26 \mathrm{ab}$ & $3,61 \mathrm{a}$ & $53,55 \mathrm{a}$ & $72,11 \mathrm{a}$ & $54,76 \mathrm{a}$ \\
\hline 100 & $31,5 \mathrm{a}$ & $1,64 \mathrm{a}$ & $3,06 \mathrm{a}$ & $12,07 \mathrm{a}$ & $3,06 \mathrm{a}$ & $3,62 \mathrm{a}$ & $48,77 \mathrm{a}$ & $64,06 \mathrm{a}$ & $62,73 \mathrm{a}$ \\
\hline $\mathrm{DMS}$ & 5,10 & 0,63 & 0,85 & 4,61 & 0,86 & 3,74 & 35,07 & 52,59 & 27,98 \\
\hline
\end{tabular}

Promedios con letras distintas indican diferencia significativa según la prueba de Tukey $(P \leq 0,05)$; DMS: diferencia mínima significativa.

que define los niveles aceptables de coliformes en alimentos de consumo humano ( $\left.10^{2} \mathrm{UFC} / \mathrm{g}\right)$. No se encontró la presencia de Salmonella ap. en ninguna de las muestras analizadas.

\section{DISCUSIÓN}

Con los resultados obtenidos es posible aprovechar los residuos sólidos orgánicos y vegetales urbanos, además, de obtener un compost de calidad para ser utilizado en la producción de hortalizas. Esto es confirmado puesto que el compost obtenido presenta excelente grado de maduración y un alto valor nutricional para la producción vegetal. Además, el proceso de compostaje de los residuos empleado demostró ser una alternativa viable.

El compost madurado presentó una tendencia general de reducción de macro, micronutrientes y metales pesados, con excepción del N, Fe, Mn y Zn, que tuvieron valores superiores en comparación a la materia prima. El beneficio del proceso de compostaje es la disponibilidad de nutrientes presentes en las materias primas para las plantas.

La pérdida de nutrientes en el proceso de descomposición de los residuos orgánicos ocurrieron, probablemente, debido a la lixiviación de los mismos, provocado por los niveles de riego necesarios para mantener la humedad del montículo. Según Valente et al. (2009), la humedad en el proceso de compostaje tiene mucha importancia en el desarrollo de los microrganismos y debe situarse entre el 50 y $60 \%$. Los compostajes con niveles inferiores al 30\% de humedad, inhiben la actividad microbiana y por encima de $65 \%$ proporciona una descomposición lenta, generada por condiciones anaerobiosis y lixiviación de nutrientes.

La relación $\mathrm{C} / \mathrm{N}$ al inicio del compostaje fue alrededor de 26/1. Según Selle (2007), para que haya equilibrio en los procesos bioquímicos de inmovilización y mineralización del $\mathrm{N}$, es necesario que la relación $\mathrm{C} / \mathrm{N}$ sea en torno al 25\%. La evaluación del carbono orgánico en el montículo de compost, mostró una reducción en el tiempo que transcurrió la descomposición de los residuos en el montículo. Probablemente, este hecho ocurrió debido al crecimiento de microrganismos que utilizaron la fuente de carbono ofertada (Mees et al, 2009), de este modo, el aumento de la actividad microbiana en los primeros días de descomposición se debe a la mayor disponibilidad de carbono en este período, así como la presencia de una fuente de materia orgánica fácilmente asimilable (Ortiz et al., 2012).

En estudios similares de compostaje, el contenido de carbono disminuyó con el progreso de la descomposición (Mees et al., 2009). Considerando que las concentraciones de $\mathrm{C}$ giran en torno al proceso de descomposición de la materia orgánica, y que a través de la actividad microbiana, los residuos orgánicos son transformados en material humificado. El compostaje de diferentes residuos orgánicos favorecieron la reducción del COT debido a la mineralización de la materia orgánica (Silva y Mendonça, 2007).

La reducción del contenido de COT también se encuentra relacionado con la fracción soluble de las fibras del material orgánico descompuesto. En el proceso de compostaje se presenta un predominio de los 
ácidos fúlvicos (AF) en relación a los ácidos húmicos $(\mathrm{AH})$, pero esto cambia al final del proceso de compostaje. El proceso de degradación de los compuestos polisacáridos y aminoácidos, permite la mayor solubilidad relativa de los AF en medio ácido y básico, proporcionando mayor desarrollo de la actividad microbiana. Estos resultados también fueron obtenidos por Silva et al. (2009), quienes analizan las fracciones del componente orgánico y observan que los ácidos fúlvicos y huminas fueron superiores a los de ácidos húmicos al final de la estabilización del compostaje.

En relación a los nutrientes aportados por el compostaje, en el trabajo de Batista y Vinhal (2010), los contenidos de $\mathrm{Ca}$, Ky N, fueron los de mayor concentración en el compost, seguidos de Fe, Zn, Mn, Cu y $\mathrm{B}$, en menores proporciones. De acuerdo con estos autores, los residuos sólidos orgánicos domiciliares (de origen doméstico), son una importante fuente de materia orgánica, proporcionando nutrientes esenciales para las plantas, los cuales pueden estar disponibles cuando el compostaje es adicionado al suelo.

Con respecto a la potencialidad del compost en la agricultura, es indispensable una rigorosa descomposición de los componentes orgánicos (materias primas) y el cumplimiento de la normatividad por el Ministerio de Agricultura (Santos et al., 2014). Sobre estos aspectos, el compost obtenido cumple con lo establecido a contaminantes biológicos, ya que fue inferior al límite permito, de esta manera faculta su utilización como abono para la producción agrícola. De igual forma, los resultados de producción de lechuga en el sistema hidropónico abierto utilizando el compost como fuente de nutrientes, permite confirmar su uso como fertilizante, ya que el compost adicionado al substrato de fibra de coco, fue capaz de producir la lechuga; pero en algunos aspectos de la producción de las plantas, no se presentaron diferencia estadística entre las dosis aplicadas. Estos resultados se deban al elevado coeficiente de variabilidad (Tab. 6 y 7), hecho que ocasionó la pérdida de sensibilidad en las pruebas utilizadas.

Las hortalizas tienen diferentes exigencias nutricionales y la toma de nutrientes difiere durante su crecimiento y desarrollo, por ejemplo plantas no fructíferas como la lechuga, presentan lenta absorción de nutrientes durante la primera mitad del ciclo de cultivo y se acelera cercano a la cosecha (Cardoso et al., 2011). De esta forma, el aumento de los nutrientes en las plantas de lechuga, se vio favorecido a mayores dosis de compost, donde probablemente no se agotaron los nutrientes.
Por otro lado, el sistema hidropónico abierto empleado no circuló solución nutritiva y el compost fue la única fuente de nutrientes para el cultivo, pero el corto ciclo del cultivo de la lechuga (35 d) limitó la liberación de nutrientes presente en el compost. La combinación del compost con la solución nutritiva puede ser una alternativa para la utilización de este sistema de producción en un primer ciclo, para que permita una oferta de nutrientes sin perjudicar la producción del cultivo.

Diversos autores han constatado en sus investigaciones que el abonamiento con compost proporciona un aumento en el rendimiento de la lechuga (Oliveira et al., 2009; Oliveira et al., 2010; Nazareno et al., 2010; Silva et al., 2011; Santana et al., 2012). Además, la literatura sobre la utilización de compostajes orgánicos como fuente única de nutrición para la lechuga en sistema hidropónico es todavía escaza. Este trabajo presenta resultados que confirma esta alternativa viable y de gran importancia para retirar residuos orgánicos de basurales y rellenos sanitarios, donde no es el destino más adecuado.

\section{CONCLUSIONES}

El compost producido a partir de residuos vegetales, orgánicos y estiércol bovino presenta las características físico-químicas y microbiológicas dentro de los estándares de utilización agronómica. El compost adicionado al sustrato de fibra de coco es capaz de favorecer la producción de plantas de lechuga. El ciclo de cultivo ( $35 \mathrm{~d}$ ) no fue suficiente para la completa liberación de los nutrientes en el sistema de producción.

Conflicto de intereses: el manuscrito fue preparado y revisado con la participación de los autores, quienes declaran no tener algún conflicto de interés que coloquen en riesgo la validez de los resultados aquí presentados.

\section{REFERENCIAS BIBLIOGRÁFICAS}

Albertin, R.M., E. Moraes, G.A. Neto, B.L.D. Angelis, E. Corveloni y F.F. Silva FF. 2010. Diagnóstico da gestão dos resíduos sólidos urbanos do município de Flórida Paraná. Rev. Agro@mbiente 4(2), 118-125. Doi: 10.18227/1982-8470ragro.v4i2.378

APHA. 2012. Standard methods for the examination of water and wastewater. Compendium of methods for the 
microbiological examination of foods. $22^{\text {th }}$ ed. American Public Health Association, Washington DC, USA.

Batista, W.D.R. y F.I.C. Vinhal. 2010. Compostagem doméstica: alternativa de aproveitamento de resíduos sólidos orgânicos. Rev. Bras. Agroecol. 5(2), 81-88.

Boer, E.D., A. Jedrczak, Z. Kowalski, J. Kulczycka y R. Szpadt. 2010. A review of municipal solid waste composition and quantities in Poland. Waste Manag. 30(3), 369-377. 10.1016/j.wasman.2009.09.018

Butu, A.W. y S.S. Mshelia. 2014. Municipal solid waste disposal and environmental issues in Kano metropolis, Nigéria. Brit. J. Env. Sci. 2(2), 10-26.

Cardoso, A.I.I., K.P. Ferreira, R.M. Vieira Júnior y C. Alcarde. 2011. Alterações em propriedades do solo adubado com composto orgânico e efeito na qualidade das sementes de alface. Hort. Bras. 29(4), 594-599. Doi: 10.1590/S0102-05362011000400025

Carmo Filho, F. y O.F. Oliveira. 1995. Mossoró: um município do semi-árido nordestino, caracterização climática e aspecto florístico. Coleção Mossoroense, série B. ESAM, Mossoró, Brasil.

Cotta, J.A.O., N.L.C. Carvalho, T.S. Brum y M.O.O. Rezende. 2015. Compostagem versus vermicompostagem: comparação das técnicas utilizando resíduos vegetais, esterco bovino e serragem. Eng. Sanit. Ambient. 20(1), 65-78. Doi: 10.1590/S1413-41522015020000111864

Dores-Silva, P.R., M.D. Landgraf y M.O.O. Rezende. 2013. Processo de estabilização de resíduos orgânicos: vermicompostagem versus compostagem. Quím. Nova 36(5), 640-645. Doi: 10.1590/S0100-40422013000500005

Embrapa. 2009. Manual de análises químicas de solo, plantas e fertilizantes. Empresa Brasileira de Pesquisa Agropecuária, Brasília, Brasil.

Figueiredo, P.G. y F.Y. Tanamati. 2010. Adubação orgânica e contaminação ambiental. Rev. Verde Agroecol. Desenvolv. Sustent. 5(3), 1-4.

Gouveia, N. 2012. Resíduos sólidos urbanos: impactos socioambientais e perspectiva de manejo sustentável com inclusão social. Ciênc. Saúde Coletiva 17, 15031510. Doi: 10.1590/S1413-81232012000600014

INMET (Instituto Nacional de Meteorologia). 2016. En: http://www.inmet.gov.br/portal/; consulta: enero de 2016

Kiehl, E.J. 2002. Manual de compostagem: maturação e qualidade do composto. $3^{\mathrm{a}} \mathrm{ed}$. Piracicaba, Brasil.

Kumar, S., S.R. Sith, G. Fowle, R.C. Velis, J. Kumar, S. Arya, R. Kumar y C. Cheeseman. 2017. Challenges and opportunities associated with waste management in India. Royal Soc. Open Sci. 4(3), 1-11. Doi: 10.1098/ rsos. 160764

Lesteur, M., V. Bellon-Maurel, C. Gonzalez, E. Latrille, J.M. Roger, G. Junqua y J.P. Steyer. 2010. Alternative methods for determining anaerobic biodegradability: A review. Process Biochem. 45(4), 431-440. Doi: 10.1016/j.procbio.2009.11.018

Li, Z., H. Lu, L. Ren y L. He. 2013. Experimental and modelling approaches for food waste composting. Chemosphere 93(7), 1247-1257. Doi: 10.1016/j. chemosphere.2013.06.064

Maragno, E.S., D.F. Trombin y E. Viana. 2007. O uso da serragem no processo de minicompostagem. Eng. Sanit. Ambient. 12(4), 355-360. Doi: 10.1590/ S1413-41522007000400001

Mees, J.B.R., S. Damasceno, M.A.V. Boas, A. Fazolo y S.C. Sampaio. 2009. Estabilização da biomassa de aguapé através da compostagem com águas resíduárias de suínos e resíduos de frigorífico. Semina: Ciênc. Agrár. 30(3), 709-716.

Montemurro, F., D. Ferri, F. Tittarelli, S. Canali y C. Vitti. 2010. Anaerobic digestate and on-farm compost application: effects on lettuce (Lactuca sativa L.) crop production and soil properties. Compost Sci. Util. 18(3), 184-193. Doi: 10.1080/1065657X.2010.10736954

Nazareno, G.G., A.M.R. Junqueira y J.R. Peixoto. 2010. Utilização de matéria orgânica para o controle de nematóides das galhas em alface sob cultivo protegido. Biosci. J. 26(4), 579-590.

Oliveira, E.M., S.B. Queiroz y V.F. Silva. 2009. Influência da matéria orgânica sobre a cultura da alface. Eng. Ambient. 6(2), 285-292.

Oliveira, E.Q., R.J. Souza, M.C.M Cruz, V.B. Marques y A.C. França. 2010. Produtividade de alface e rúcula, em sistema consorciado, sob adubação orgânica e mineral. Hort. Bras. 28(1), 36-40. Doi: 10.1590/ S0102-05362010000100007

Ortiz, P.L.G., R.O. Lizaola, V.E. Hernández, G.S.B. Valdés y M.J.S. Colín. 2012. Respiración de $\mathrm{CO}_{2}$ como indicador de la actividad microbiana en abonos orgánicos de lupinus. Terra Latinoam. 30(4), 355-362.

Pedrosa, T.D., C.A.S. Farias, R.A. Pereira y E.T.R. Farias. 2013. Monitoramento dos parâmetros físico-químicos na compostagem de resíduos agroindustriais. Nativa 1(1), 44-48. Doi: 10.14583/2318-7670.v01n01a08

Pereira Neto, J.T. 2007. Manual de compostagem: processo de baixo custo. UFV, Viçosa-MG, Brasil.

Rezende, J.H. 2013. Composição gravimétrica e peso específico dos resíduos sólidos urbanos em Jaú (SP). Eng. Sanit. Ambient. 18(1), 1-8. Doi: 10.1590/ S1413-41522013000100001

Santana, C.T.C., A. Santi, R. Dallacort, M.L. Santos y C.B. Menezes. 2012. Desempenho de cultivares de alface americana em resposta na diferentes doses de torta de filtro. Rev. Ciênc. Agron. 43(1), 22-29. Doi: 10.1590/ S1806-66902012000100003 
Santos, C.S., N.S. Henrique, J.A. Shhlinder, E. Ferreira y R. Stachiw. 2014. Aproveitamento da fração orgânica dos resíduos sólidos urbanos para produção de composto orgânico. Rev. Bras. Ciênc. Amaz. 3(1), 15-28.

Selle, G.L. 2007. Ciclagem de nutrientes em ecossistemas florestais. Biosc. J. 23(4), 29-39.

Silva, E.M.N.C.P., R.L.F. Ferreira, S.E. Araújo Neto, L.B. Tavella y A.J.S. Solino. 2011. Qualidade de alface crespa cultivada em sistema orgânico, convencional e hidropônico. Hort. Bras. 29(2), 242-245. Doi: 10.1590/ S0102-05362011000200019

Silva, F.A.M., F.G. Lopez, R.L Villas Boas y R.B. Silva. 2009. Transformação da matéria orgânica em substâncias húmicas durante a compostagem de resíduos vegetais. Rev. Bras. Agroecol. 4(1), 59-66.

Silva, I.R. y E.S. Mendonça. 2007. Matéria orgânica do solo. pp. 275-374. En: Novais, R.F., V.H. Alvarez, N.F.
Barros, R.L.F. Fontes, R.B. Cantarutti y J.C.L. Neves (eds.). Fertilidade do solo. Sociedade Brasileira de Ciência do Solo, Viçosa-MG, Brasil.

Srivastava, V., S.A. Ismail, P. Singh y R.P. Singh. 2015. Urban solid waste management in the developing world with emphasis on India: challenges and opportunities. Rev. Env. Sci. Bio/Tec. 14(2), 317-337. Doi: 10.1007/ s11157-014-9352-4

Valente, B.S., E.G. Xavier, T.B.G.A. Morselli, D.S. Jahnke, B.S. Brum Junior, B.R. Cabrera, P.O. Moraes y D.C.N. Lopes. 2009. Fatores que afetam o desenvolvimento da compostagem de resíduos orgânicos. Arch. Zootec. 58(1), 59-85.

Yu, H. y G.H. Huang. 2009. Effects of sodium acetate as a $\mathrm{pH}$ control amendment on the composting of food waste. Bioresour. Technol. 100(6), 2005-2011. Doi: 10.1016/j.biortech.2008.10.007 